\title{
Beschreibung und Evaluation eines Manuals für Herzratenvariabilitäts-Biofeedback bei der somatischen Belastungsstörung
}

\author{
Laura Klewinghaus Alexandra Martin \\ Abteilung für Klinische Psychologie und Psychotherapie, Bergische Universität Wuppertal, Wuppertal, Deutschland
}

\section{Schlüsselwörter}

Herzratenvariabilität · Biofeedback · Intervention ·

Somatische Belastungsstörung · Psychophysiologie

\begin{abstract}
Zusammenfassung
Hintergrund: Herzratenvariabilitäts-Biofeedback (HRV-BF) zeigt erste Wirksamkeitshinweise bei Depressionen, Angststörungen oder funktionellen somatischen Syndromen. Bei der somatischen Belastungsstörung (SBS) stehen diese noch aus. Ziel der vorliegenden Arbeit war es, eine neu entwickelte HRV-BF-Kurzintervention vorzustellen und die HRV detailliert zu analysieren sowie die Anwendbarkeit und Akzeptanz bei der SBS zu überprüfen. Methoden: Analysiert wurden hier die Daten der Teilstichprobe einer randomisiert kontrollierten Pilotstudie (22 Personen mit SBS nach DSM-5), die ein HRV-BF über 4 Sitzungen erhielt. Erhoben wurden die HRV (SDNN: "standard deviation of the NN interval", RMSSD: "root mean square of successive differences between NN interval", LF: "low frequency"), die Bewertung und Akzeptanz der Intervention auf subjektiver Ebene. Ergebnisse: HRVAnalysen innerhalb der Therapiesitzungen zeigten, dass die Personen lernten, ihre HRV signifikant während der Biofeedbackeinheiten zu erhöhen und diese in Selbstregulationsphasen ohne Feedback aufrechtzuerhalten (SDNN, RMSSD, LF: $\left.5,7 \leq F_{t} \leq 11,1\right)$. Zudem zeigte sich eine Verbesserung der HRV über die Sitzungen hinweg (SDNN). Die Intervention wurde durch die Mehrheit der Teilnehmer*innen sehr positiv bewertet (u.a. Zufriedenheit, Verbesserung der Stimmung und des körperlichen Befindens). Schlussfolgerungen: HRV-BF kann in 4 Sitzungen gut erlernt werden und
\end{abstract}

zeigt positive Effekte bei Patient*innen mit SBS. Die Hinzunahme des HRV-BF zu bestehenden Interventionen, z.B. Psychotherapie, scheint vielversprechend.

(c) 2022 The Author(s).

Published by S. Karger AG, Basel

\author{
Presentation and Evaluation of a Manual for Heart \\ Rate Variability Biofeedback in Somatic Symptom \\ Disorder
}

\section{Keywords}

Heart rate variability · Biofeedback · Intervention · Somatic symptom disorder · Psychophysiology

\section{Abstract}

Background: There is initial evidence for the efficacy of heart rate variability biofeedback (HRV-BF) in depression, anxiety disorders and functional somatic syndromes. In somatic symptom disorder (SSD) evidence is lacking. The aim of the study was to describe a newly developed HRVBF brief intervention and to analyze HRV changes, and to examine the applicability and acceptance in SSD. Methods: We analyzed the data of the subsample of a pilot randomized controlled trial (22 subjects with SSD) who received HRV-BF over 4 sessions. We assessed HRV (SDNN: standard deviation of the NN interval, RMSSD: root mean square of successive differences between NN interval, LF: low frequency), and the subjective evaluation and acceptance of the intervention. Results: HRV analyses within
Karger@karger.com www.karger.com/ver

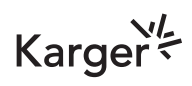

BOPEN ACCESS (c) 2022 The Author(s)

Published by S. Karger AG, Basel

This article is licensed under the Creative Commons Attribution 4.0 International License (CC BY) (http://www.karger.com/Services/ OpenAccessLicense). Usage, derivative works and distribution are permitted provided that proper credit is given to the author and the original publisher.
Korrespondenz an:

Laura Klewinghaus, klewinghaus@uni-wuppertal.de 
therapy sessions showed that individuals learned to in crease their HRV significantly during biofeedback sessions and were able to maintain it during self-regulation periods without feedback (SDNN, RMSSD, LF: $5.7 \leq F_{t} \leq$ 11.1). Moreover, HRV improved across sessions (SDNN). The majority of participants rated the intervention very positively (e.g., satisfaction, improvement in mood and physical well-being). Conclusions: HRV-BF can be learned within 4 sessions and shows positive effects in patients with SSD. Adding HRV-BF to existing treatments, e.g. psy chotherapy, seems promising.

(c) 2022 The Author(s). Published by S. Karger AG, Basel

\section{Theoretischer Hintergrund}

Die Herzratenvariabilität (HRV) kann als Index für den Gesundheitszustand eines Individuums angesehen werden [Appelhans und Luecken, 2006]. Sie zeigt das Zusammenspiel der sympathischen und parasympathischen Einflüsse auf die Herzrate an und kann als Messgröße für die Funktionalität des autonomen Nervensystems betrachtet werden [Appelhans und Luecken, 2006]. Eine hohe HRV kennzeichnet, dass eine Person sich adaptiv und flexibel regulieren kann [Thayer et al., 2012]. Eine niedrige HRV hingegen ist mit einer sympathovagalen Dysbalance assoziiert und geht mit Erkrankungen (z.B. Diabetes oder kardiovaskuläre Erkrankungen) und einem erhöhten Mortalitätsrisiko einher [Appelhans und Luecken, 2006; Thayer et al., 2012]. Neben dem körperlichen Wohlbefinden wird sie auch als Marker für emotionales Wohlbefinden und Emotionsregulation angesehen [Appelhans und Luecken, 2006; Mather und Thayer, 2018]. Von Beauchaine und Thayer [2015] wird die HRV als transdiagnostischer Marker für Psychopathologie angesehen. Meta-Analysen zeigten beispielsweise eine reduzierte HRV bei depressiven Störungen und Angststörungen [Chalmers et al., 2014; Koch et al., 2019]. Auch bei anderen psychischen Störungen kann von einer autonomen Dysbalance ausgegangen werden [Quintana et al., 2013; Sammito et al., 2015].

Eine Interventionsmöglichkeit, die direkt an der sympathovagalen Dysbalance ansetzt, indem die HRV erhöht wird, stellt das HRV-Biofeedback (HRV-BF) dar [Lehrer und Gevirtz, 2014]. Neben der Wiederherstellung der Regulationsfähigkeit des autonomen Nervensystems hat es positive Effekte auf den Vagusnerv und stimuliert entzündungshemmende Vorgänge [Lehrer und Gevirtz, 2014]. Biofeedback ist eine Behandlungsmethode, bei der Körpersignale über einen Bildschirm den Patient*innen zurückgemeldet werden, die mit der Psychopathologie assoziiert sind [Rief und Birbaumer, 2011]. Primär sollen die Patient*innen lernen, ihre Körpersignale zu beein- flussen und wieder eine Kontrolle über ihre Körperfunktionen zu erlernen. Neben den zuvor genannten Wirkmechanismen, ist anzunehmen, dass auch psychologische Lerneffekte wie eine Steigerung der Selbstwirksamkeit und Kontrollüberzeugungen bedeutend sind [Holroyd et al., 1984]. Beim HRV-BF im Speziellen werden den Patient*innen die Herzrate und Atmung als Feedbacksignale rückgemeldet und mit einem Taktgeber die Atemfrequenz angezeigt, in der die Patient*innen atmen sollen. Sie sollen erlernen, ihre Herzrate durch die Atmung zu beeinflussen. Es werden zwei Arten des HRV-BF unterschieden: Entweder mit der Vorgabe von einer Atemfrequenz mit z.B. 6 Atemzügen pro Minute $(0,1 \mathrm{~Hz})$ oder als Resonanzfrequenzbiofeedback (RF-BF). Bei Letzteren hat die respiratorische Sinusarrhythmie eine zentrale Rolle, die die rhythmischen Schwankungen der Herzrate durch die Atmung darstellt [Lehrer und Gevirtz, 2014]. Die HRV ist am höchsten, wenn Herzrate und Atmung parallel verlaufen. Die Atemfrequenz, bei der die höchste Übereinstimmung der Herzrate und Atemfrequenz besteht, wird als Resonanzfrequenz (RF) bezeichnet. Beim RF-BF wird die individuelle RF eines*r Patient*in ermittelt und das Biofeedback damit durchgeführt. Es hat sich gezeigt, dass das HRV-BF auf Basis der individuell bestimmten RF zu besseren Ergebnissen führt als das Vorgehen mit fester Vorgabe der Atemfrequenz [Steffen et al., 2017]. Lehrer und Kollegen entwickelten zum RFBF ein Manual mit 10 Sitzungen und verkürzten dieses im Verlauf auf 5 Sitzungen [Lehrer et al., 2000, 2013]. Evidenz für die Wirksamkeit des HRV-BF und im Speziellen des RF-BF liegt bei verschiedenen Störungen bereits vor [Lehrer et al., 2020]. Ein Review von Schmidt und Martin [2017] zeigte positive Effekte durch das HRV-BF sowohl bei körperlichen (u.a. kardiovaskuläre Störungen, funktionelle Syndrome) als auch psychischen (u.a. posttraumatische Belastungsstörung; Substanzabhängigkeit) Erkrankungen. Die Effekte des HRV-BF hinsichtlich somatoformer Störungen und der im DSM-5 eingeführten somatischen Belastungsstörung (SBS) sind jedoch bislang nicht untersucht.

Die SBS ist gekennzeichnet durch eines oder mehrere körperliche Symptome und geht mit psychobehavioralen Auffälligkeiten (exzessive Gedanken, Verhalten und Gefühle im Zusammenhang mit den körperlichen Beschwerden) einher [APA, 2013]. Erste Studien legen auch bei diesem Störungsbild eine verringerte HRV im Vergleich zu gesunden Personen nahe [Cheng et al., 2020]. Sowohl die Leitlinie für funktionelle Körperbeschwerden [AWMF, 2018] als auch die Leitlinie zur Psychotherapie somatoformer Störungen [Martin et al., 2013] empfehlen Biofeedback im Rahmen einer gestuften Behandlung. Erste Studien legen positive Effekte des HRV-BF bei funktionellen somatischen Syndromen (z.B. Reizdarmsyndrom, Fibromyalgie) oder Schmerzsyndromen (z.B. 
chronische Nackenschmerzen) in Form einer verbesserten Lebensqualität und einer Verringerung der Symptome nach der Intervention nahe [Ryan und Gevirtz, 2004; Schmidt et al., 2012; Windthorst et al., 2017]. Aufgrund der angenommenen Parallelen der SBS mit funktionellen somatischen Syndromen und der zuvor beschriebenen Befunde ist anzunehmen, dass das HRV-BF auch für die SBS wirksam sein wird. Es lässt sich vermuten, dass es als Kurzintervention geeignet ist, da die Kontrolle über die Körperfunktionen schnell erworben werden kann. Eine Studie konnte bereits nach einer HRVBF-Sitzung und vierwöchigem Training zu Hause eine Verbesserung der Symptomatik bei Personen mit posttraumatischer Belastungsstörung erzielen [Schuman und Killian, 2019]. Zudem berichteten Hassett et al. [2007], dass Patient*innen mit Fibromyalgie lernen, ihre HRV während der Biofeedbacksitzungen zu erhöhen. Allgemein evaluieren nur wenige HRV-BF-Studien die Veränderung der HRV, ihre Anwendbarkeit sowie Benutzerakzeptanz.

Eine HRV-BF-Kurzintervention bietet eine gute Ergänzung zu einer kognitiv-verhaltenstherapeutischen Psychotherapie [Lehrer, 2018]. Durch das HRV-BF können physiologische Aspekte der psychischen Erkrankungen wie z.B. SBS oder Panikstörung direkt angesprochen werden. Entspannungsverfahren sind zwar schon gut in der psychotherapeutischen Behandlung etabliert, sprechen aber nur die sympathische Aktivierung an [Lehrer, 2018]. Das HRV-BF hat den Vorteil, die autonome Aktivität zu modulieren und wirkt über das parasympathische vagale System. Im Bereich der somatoformen Störung bzw. SBS besteht die Notwendigkeit der Weiterentwicklung psychologischer Interventionen, da die Effektstärken bestehender Interventionen nur klein bis moderat sind [van Dessel et al., 2014] und Patient*innen oftmals lange (ca. 25 Jahre) benötigen, bis sie eine adäquate Behandlung erhalten [Herzog et al., 2018]. Zusammenfassend stellt HRV-BF eine vielversprechende Therapiemöglichkeit dar, die aber noch weiterer Forschung bedarf.

Die vorliegende Arbeit ist Teil einer randomisiertkontrollierten Studie zur Wirksamkeit von HRV-BF bei SBS. Ziel des vorliegenden Artikels ist es, das entwickelte Behandlungsmanual (HRV-BF als Kurzintervention mit 4 Sitzungen) genauer darzustellen und in seiner Anwendbarkeit und Akzeptanz am Störungsbild der SBS zu überprüfen. Zum einen soll überprüft werden, ob die Personen während der Trainingssitzungen das Prinzip des HRV-BF erlernen, indem die physiologischen Veränderungen detailliert betrachtet werden. Dabei wird eine Steigerung der HRV im Verlauf der Biofeedbacksitzung angenommen. In der ersten und zweiten Sitzung liegt der Fokus vor allem auf der Bestimmung der Resonanzfrequenz und der Einübung von Atemtechniken, so dass sich die Fragestellungen auf die letzten beiden Sitzungen beziehen, in denen das klassische HRV-BF eingeübt wird. Zudem wird überprüft, ob die Intervention übergeordnet zu einer Veränderung der HRV während der Ruhemessungen über die Sitzungen hinweg beiträgt. Die Akzep$\operatorname{tanz}$ der Intervention wird auf subjektiver Ebene überprüft.

\section{Methode: Darstellung des Manuals}

\section{Aufbau und Konzept der Kurzintervention}

Die hier eingesetzte Kurzintervention eines HRV-BF wurde in Anlehnung an das Manual zum RF-BF von Lehrer et al. [2013] entwickelt und umfasst vier Sitzungen (siehe Online-Supplement-Material S1, verfügbar unter www.karger.com/doi/10.1159/000522175). Die Patient*innen erhalten zum einen psychoedukative Informationen über das Störungsbild und zum anderen ein RF-BF, welches um Hausaufgaben mit einer App (siehe Abschnitt Sitzung 1) ergänzt wird. Im Folgenden werden die vier Sitzungen jeweils beschrieben. Während der Trainingssitzungen erfolgt zu Beginn eine kurze Rückschau auf die vergangene Woche, und es werden die zu Hause durchgeführten Übungen besprochen. Am Ende jeder Sitzung erfolgt gemeinsam mit den Patient*innen eine Evaluation der Sitzung mit möglichen Problemen und bemerkten körperlichen Veränderungen während der Übungen.

Sitzung 1. In der ersten Sitzung liegt der Fokus auf der Psychoedukation und auf der Bestimmung der Resonanzfrequenz. Den Patient*innen wird eine Beschreibung der HRV gegeben, und die Zusammenhänge zu ihren Beschwerden werden deutlich gemacht. Es wird auf Studienbefunde verwiesen, die zeigen, dass die HRV ein Indikator für den Gesundheitszustand ist und bei chronischen Schmerzen oder Körperbeschwerden herabgesetzt sein kann. Den Patient*innen wird dann die respiratorische Sinusarrhythmie und das Rational des Biofeedbacks erklärt. Dies wird in das biopsychosoziale Modell [Engel, 1977], das an chronische Körperbeschwerden angepasst wurde, eingeordnet. Im zweiten Abschnitt der Sitzung werden den Patient*innen die Sensoren zur Ableitung der Atmung (dehnungssensibler Atemgurt) und der Herzrate (3-Kanal-EKG) angelegt. Nach einer Adaptationsphase (etwa $2 \mathrm{~min}$ ), erfolgt eine Ruhemessung (etwa $5 \mathrm{~min}$ ), um die Ausgangswerte zu bestimmen. Die Patient*innen sollen dafür eine entspannte Körperhaltung einnehmen und in Ruhe atmen. Der nächste Teil der Sitzung dient der Bestimmung der RF (die höchste Übereinstimmung der Herzrate und Atemfrequenz). Den Patient*innen werden die verschiedenen Kurven (Herzrate, Atemkurve, Hilfslinie/Taktgeber) am Bildschirm erklärt. Danach werden die Patient*innen instruiert, in fünf verschiedenen Atemfrequenzen zu atmen, wobei ein 
Taktgeber der Orientierung dient: "Atmen Sie nun in der Frequenz, wie es der Taktgeber vorgibt. Atmen Sie ein, wenn die Linie hochgeht und aus, wenn die Linie wieder runtergeht." Der*die Therapeut*in weist darauf hin, dass die Patient*innen nicht zu tief einatmen sollen, um Symptome der Hyperventilation $\mathrm{zu}$ vermeiden. Den Patient*innen werden nacheinander die fünf verschiedenen Atemfrequenzen 6 Atemzüge pro Minute (breath per minute, bpm), 5,5, 5, 4,5 und 6,5 bpm für jeweils 4 min vorgegeben und mit 1 min Pause gewechselt. Bis zur nächsten Sitzung wird dann die RF anhand verschiedener HRV-Parameter (Peak bei 0,1 Hz; maximale Low-frequency-Power (LF)) bestimmt. Im Anschluss an die Sitzung wird den Patient*innen die Hausaufgabe erklärt, in der die Patient*innen mit Hilfe einer App "Breathball" (https://breathball.com) zweimal täglich für $10 \mathrm{~min}$ atmen sollen. Die Hausaufgaben dienen dazu, das Erlernte in den Alltag zu übertragen. Die App besitzt einen individuell einstellbaren visuellen Taktgeber für die Atemfrequenz, aber ohne Biofeedback- und Protokollfunktion. Als Einstieg für die oftmals ungewohnte langsame Atmung wird dieser Taktgeber nach der ersten Sitzung auf $6 \mathrm{bpm}$ eingestellt und nach der zweiten Sitzung, wenn die RF bestimmt wurde, auf diese angepasst. Zudem werden die Patient*innen angeleitet, einen Protokollbogen zur Dokumentation der Hausaufgaben zu führen, in denen sie notieren, wann und wie lange sie die Übungen durchgeführt haben und reflektieren, wie sie diese erlebt haben.

Sitzung 2. Diese Sitzung dient der Feinabstimmung der RF und der Einführung verschiedener Atemtechniken. $\mathrm{Zu}$ Beginn werden die Patient*innen gebeten, in der bislang ermittelten RF mit Taktgeber wie in der vorherigen Sitzung zu atmen. Zudem erfolgt das Atmen in einem halben Atemzug mehr und weniger als der RF. Auch hier erfolgt das Atmen für 4 min mit jeweils 1 min Pause. Die RF wird während der Sitzung final bestimmt und im weiteren Verlauf als Taktgeber eingestellt. Der zweite Abschnitt der Sitzung dient der Erklärung der Lippenbremsenatmung und der tiefen Bauchatmung. Zunächst werden die Techniken ohne den Einsatz des Biofeedbacks kurz geübt. Im Anschluss erfolgt nacheinander der Einsatz der Techniken mit der ermittelten RF anhand der Rückmeldung auf dem Bildschirm. Der*die Therapeut*in achtet während der Sitzung auf die korrekte Durchführung der Atemtechnik und Atemfrequenz und unterstützt ggf. die Patient*innen. Am Ende der Sitzung wird den Patient*innen ihre RF mitgeteilt, damit sie nun in dieser Frequenz täglich üben.

Sitzung 3. In der dritten Sitzung wird das klassische Biofeedback eingeführt. $\mathrm{Zu}$ Beginn werden die Atemtechniken der letzten Sitzung wiederholt und mögliche Probleme besprochen. Danach wird den Patient*innen erklärt, dass sie die HRV erhöhen können, indem sie im Einklang mit der Herzrate atmen und die Atemtechniken dabei anwenden. Hierzu wird zum Einstieg der Taktgeber mit der RF noch eingeblendet (Biofeedback mit Taktgeber; BF-T), jedoch nach 5 min Übungszeit ausgeblendet und die Übung wird für weitere 5 min fortgeführt (Biofeedback ohne Taktgeber; BF-1). Während der Sitzung achtet der*die Therapeut*in auf die Atmung der Patient*innen und gibt Hinweise, falls die Herzrate und Atmung nicht im Einklang sind. Das HRV-BF ohne Taktgeber wird weitere zwei Mal für 5 min (Biofeedback ohne Taktgeber; BF-2 \& BF-3) wiederholt. Die Übung wird mit einer Selbstregulationsphase (SR) von zwei Minuten beendet. Während der Selbstregulationsphase wird der Bildschirm ausgestellt und die Patient*innen werden gebeten, die Atmung aufrechtzuerhalten ohne dass sie ein Feedbacksignal erhalten.

Sitzung 4. Die letzte Sitzung dient der Wiederholung des Biofeedbacks aus Sitzung drei. Auch hier erfolgt eine Wiederholung der Atemtechniken, sowie des Biofeedbacks mit Taktgeber (BF-T). Danach erfolgen zwei Übungsblöcke von je fünf Minuten, in denen das Biofeedback ohne Taktgeber geübt wird (Biofeedback ohne Taktgeber; BF-2 und BF-3). Die Selbstregulationsphase (SR) schließt auch hier wieder an die letzte Übungseinheit an und umfasst diesmal $5 \mathrm{~min}$. Am Ende wird das Training besprochen, sowie evaluiert und den Patient*innen erklärt, dass das Fortführen der Übungen auch nach der Kurzintervention wichtig ist. Zur Verbesserung des Transfers erhalten die Patient*innen ein Arbeitsblatt, auf dem sie sich Erfahrungen sowie Perspektiven der zukünftigen Umsetzung der gelernten Übungen notieren.

\section{Methode: Überprüfung der Anwendbarkeit der Intervention}

\section{Ablauf}

Die vorliegende Arbeit stellt eine Teilanalyse einer randomisiert kontrollierten Pilotstudie dar, deren Hauptergebnisse insbesondere zum Zwischengruppenvergleich einer anderen Publikation zu entnehmen sind [Klewinghaus und Martin, eingereicht]. Die Rekrutierung der Teilnehmer*innen erfolgte in Arzt- und Physiotherapiepraxen, Schmerz-Kliniken, der Universitätsambulanz für Psychotherapie der Bergischen Universität Wuppertal sowie durch Zeitungsartikel, soziale Medien und Annoncen. Folgende Einschlusskriterien wurden vorausgesetzt: 1. das Vorliegen einer somatischen Belastungsstörung nach DSM-5, 2. mindestens zwei Symptombereiche, 3. Alter 18-65 Jahre. Ausschlusskriterien der Studie waren aufgrund bekannter Effekte auf die HRV folgende: Herzschrittmacher, akute Herzerkrankung, keine stabile Dosierung der Herz- und Psychopharmaka-Medikation (letzte 4 Wochen), BMI >35, Diabetes, Rheuma oder psychotische Symptome in der 
Abb. 1. Flussdiagramm für die HRV-BFInterventionsgruppe.

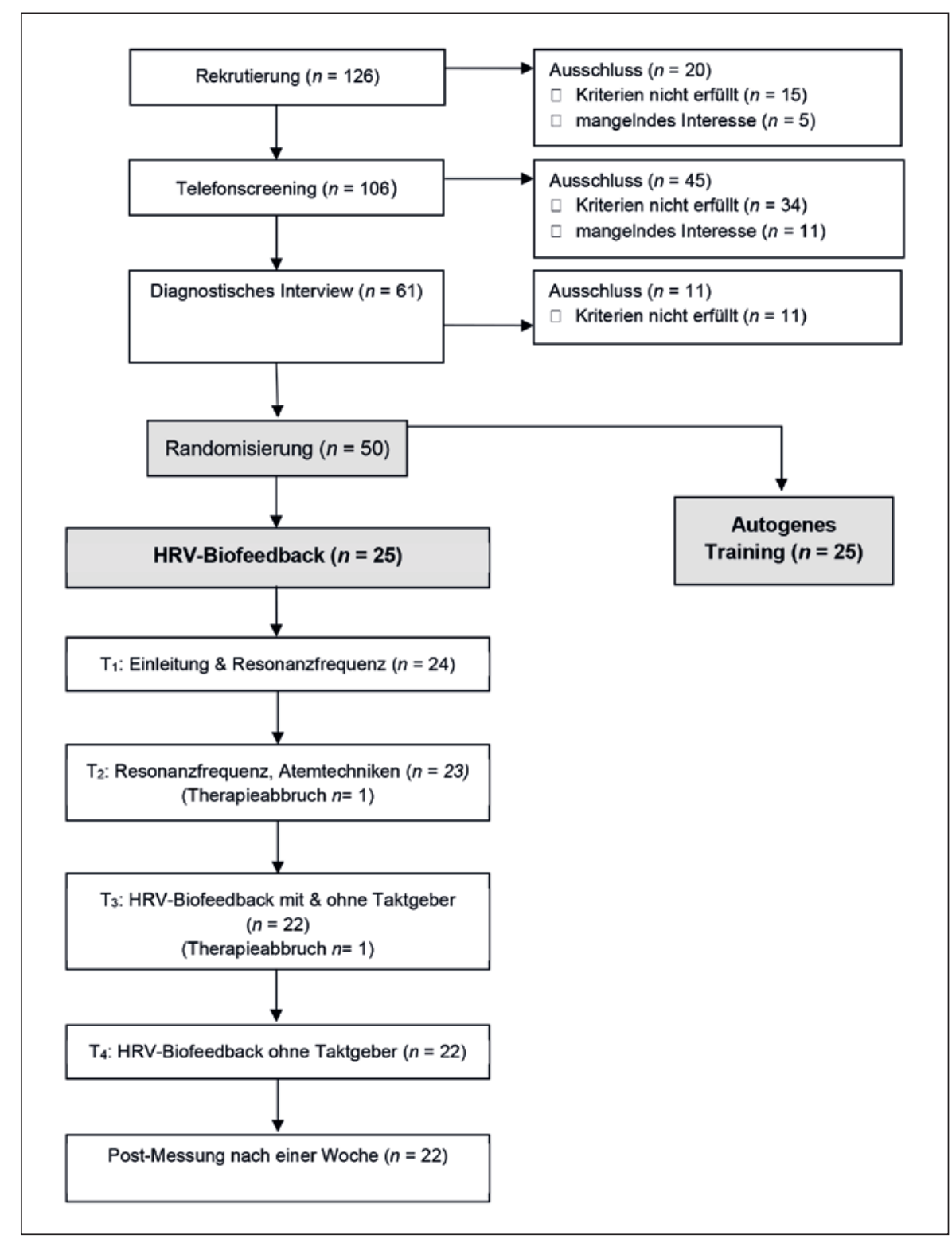

Vorgeschichte. Zu Beginn erfolgte mit interessierten Personen ein Screening am Telefon, in dem erste Einschlusskriterien überprüft und Informationen zur Studie gegeben wurden. Geeignete Personen wurden dann in die Räume der Universität Wuppertal eingeladen. Die Personen wurden über die Ziele und den Ablauf der Studie aufgeklärt und gaben ihr schriftliches Einverständnis. Es erfolgte eine diagnostische Abklärung mit einem Interview für die SBS angelehnt an die Kriterien des DSM-5 [Toussaint et al., 2019], sowie eine Abklärung von Komorbiditäten mit dem halbstrukturierten Interview Mini-Dips für DSM-5 [Margraf und Cwik, 2017]. Geeignete Personen wurden im Rahmen der Pilotstudie randomisiert in Blöcken (random.org) auf eine der Interventionsbedingungen (HRV-BF oder autogenes Training) zugeteilt und erhielten einmal wöchentlich eine Interventionssitzung. In der vorliegenden Arbeit wird nur die
Stichprobe der HRV-BF-Intervention betrachtet. Die Studie wurde im Deutschen Register Klinischer Studien (DRKS00017099) registriert, und es liegt ein positives Ethikvotum der Ethikkommission der Bergischen Universität Wuppertal (MS/BBL 190327) vor.

\section{Stichprobe}

Im Erhebungszeitraum von März 2019 bis Februar 2021 wurden 50 Personen randomisiert auf die beiden Interventionsarme HRV-BF und autogenes Training verteilt. In der vorliegenden Arbeit wird nur die Teilstichprobe betrachtet, die das HRV-BF absolvierte. Diese starteten mit $n=24$, und $n=22$ Personen schlossen die Intervention ab. Abbildung 1 ist das detaillierte Flussdiagramm für die HRV-BF-Gruppe zu entnehmen. 


\section{Messinstrumente}

Für die vorliegende Studie sind sowohl psychophysiologische Maße (HRV) als auch die subjektive Akzeptanz und Zufriedenheit mit der Intervention von Interesse. Jede Sitzung wurde vor und nach der Intervention die HRV als Ruhemessung (Baseline, BL) für 5 min und im Verlauf während der Trainingssitzungen (BF-T, BF-1, BF-2, BF-3, SR) erhoben. Die Erfassung erfolgte mittels eines 3-Kanal-EKG (1024 Abtastrate) mit dem NEXUS-4 mit der Software BioTrace Version 2018A1 (Mind Media BV). Eine Elektrode wurde unter den linken Rippenbogen und eine am Ende des rechten Schlüsselbeins platziert. Die Erdungs-Elektrode wurde an der Außenseite des rechten Arms befestigt. EKG-Rohdaten wurden extrahiert und mithilfe der Software Kubios [Tarvainen et al., 2014] analysiert. Die Sitzungsdaten wurden segmentiert und nach automatischer Artefaktkorrekur durch Kubios zusätzlich visuell überprüft. Berechnet wurden sowohl zeit- (SDNN, "standard deviation of the NN interval"; RMSSD, "root mean square of successive differences between NN interval") als auch frequenzbasierte (LF) HRV-Parameter. Ein dehnungssensibler Atemgurt mit einer Abtastrate von 32 Stichproben pro Minute diente der Erfassung der Atemfrequenz.

Zur Erfassung der Akzeptanz und Zufriedenheit wurde ein Evaluationsfragebogen, orientiert an Schmidt und Martin [2015], entwickelt, der den Umgang mit den körperlichen Symptomen, das Befinden, die Stimmung, das Stressempfinden, die Zufriedenheit mit der Intervention, die Fortführung im Alltag und Nebenwirkungen auf einer 5-Punkt-Likert-Skala (keine bis volle Zustimmung) erfasst (siehe Online-Supplement-Material S2). Diese Erhebung erfolgte eine Woche nach der letzten Sitzung in den Räumen der Universität Wuppertal im Online-Format.

Zur Erfassung multidimensionaler Therapieergebnisse wurden neben dem Screening für somatoforme Störungen [SOMS-7T; Rief und Hiller, 2008] weitere störungsspezifische Variablen sowie psychobehaviorale Aspekte und Folgen der Erkrankung gemäß den Empfehlungen von Rief et al. [2017] erfasst. Eine detaillierte Auflistung der Messinstrumente kann dem Deutschen Register Klinischer Studien entnommen werden. Diese sind nicht Bestandteil der statistischen Analysen der vorliegenden Arbeit.

\section{Statistische Auswertung}

Die statistische Auswertung erfolgte mittels SPSS Statistics 27. Zur Überprüfung der objektiven Anwendbarkeit wurden für die HRV-Parameter zur Betrachtung der Sitzungsverläufe messwiederholte einfaktorielle ANOVAs mit Kontrasten für die dritte und vierte Sitzung, in denen das klassische HRV-BF Anwendung fand, berechnet. Zur Überprüfung der Steigerung der HRV während der Sitzung wurden die Ruhemessungen (BL) mit den Biofeedbackeinheiten (BF) und der Selbstregulationsphase (SR) in den HRV-Parametern verglichen. $\mathrm{Zu}$ dem wurden messwiederholte ANOVAs für die Ruhemessungen (BL) über die vier Sitzungen hinweg berechnet. Das Signifikanzniveau wurde auf $p=0,5$ gesetzt und hinsichtlich der Post-hoc-Tests Bonferroni korrigiert. Bezüglich der Häufigkeiten des Evaluationsfragebogens wurden die Antwortmöglichkeiten "ziemlich", "stark" und "sehr stark" für eine übersichtlichere Darstellung kumuliert berichtet und als Zustimmung gewertet.

\section{Ergebnisse}

\section{Stichprobenbeschreibung}

Die 22 Personen der HRV-BF-Intervention waren im Mittel 45,2 Jahre alt (SD $=13,3)$ und zu 80\% weiblich. Die Mehrheit (57,2\%) war berufstätig, 19\% in Ausbildung und 23,8\% erwerbsunfähig oder arbeitslos. Die meisten Personen waren verheiratet oder in einer Partnerschaft (57,2\%), 19\% waren ledig, sowie $23,8 \%$ geschieden. Die Symptomatik der SBS bestand bei den Personen im Mittel 8,9 Jahre (SD = 9,4). Der Summenscore des SOMS-7 lag im Mittel bei 29,1 (SD = 15,9). Die Mehrheit der Patient*innen war arbeitsfähig $(85,7 \%)$, und $28,6 \%$ wiesen eine Komorbidität mit einer weiteren psychischen Störung auf.

\section{Objektive Anwendbarkeit}

$H R V$-Analysen innerhalb der Sitzungen. Überprüft werden sollte die Annahme einer Steigerung der HRVParameter in den letzten beiden Sitzungen von der Ruhemessung $\mathrm{zu}$ den Biofeedbackeinheiten und der Selbstregulationsphase. Die deskriptiven Daten und Teststatistiken der ANOVA sind Tabelle 1, die Post-hocAnalysen Tabelle $2 \mathrm{zu}$ entnehmen. Zur dritten Trainingssitzung zeigten sich signifikante Zeiteffekte für die HRV-Parameter SDNN, RMSSD und LF ( $p$-Werte $<0,001)$. Die Post-hoc-Analysen zeigten ebenfalls signifikante Unterschiede zwischen der Ruhemessung (BL) und den Biofeedbackeinheiten (BF-P, BF-1, BF-2 und BF-3) in den Parametern RMSSD, SDNN und LF ( $p$-Werte $<0,001)$ mit großen Effekten. Dies zeigt eine größere HRV während der Biofeedbackeinheiten im Vergleich zur Ruhemessung. Es ergab sich kein signifikanter Unterschied zwischen den Biofeedbackeinheiten (BF-P, BF-1, BF-2 und BF-3) und der Selbstregulationsphase (SR) ( $p$ Werte $>0,05$ ), während die Parameter eine größere HRV während der Selbstregulationsphase gegenüber der Ruhemessung abbilden.

Hinsichtlich der letzten Trainingssitzung ergaben sich signifikante Zeiteffekte für die HRV-Parameter SDNN, RMSSD und LF ( $p$-Werte $<0,001)$ mit großen Effekten. Die Post-hoc-Analysen zeigten einen signifikanten Un- 
Tabelle 1. HRV-Parameter der Messphasen der dritten und vierten Interventionssitzung mit ANOVA-Teststatistiken $(n=20)$

\begin{tabular}{|c|c|c|c|c|c|c|c|c|}
\hline & & $\begin{array}{l}\mathrm{BL} \\
\mathrm{M}(\mathrm{SD})\end{array}$ & $\begin{array}{l}\text { BF-T } \\
M(S D)\end{array}$ & $\begin{array}{l}B F-1 \\
M(S D)\end{array}$ & $\begin{array}{l}B F-2 \\
M(S D)\end{array}$ & $\begin{array}{l}B F-3 \\
M(S D)\end{array}$ & $\begin{array}{l}\text { SR } \\
M(S D)\end{array}$ & ANOVA-Teststatistik \\
\hline RMSSD, ms & $\begin{array}{l}\text { T3 } \\
\text { T4 }\end{array}$ & $\begin{array}{l}25,57(13,02) \\
28,19(12,97)\end{array}$ & $\begin{array}{l}35,06(15,78) \\
36,42(14,08)\end{array}$ & $34,35(17,48)$ & $\begin{array}{l}37,39(20,26) \\
36,97(13,24)\end{array}$ & $\begin{array}{l}39,46(22,85) \\
38,80(14,06)\end{array}$ & $\begin{array}{l}36,61(20,53) \\
38,33(18,54)\end{array}$ & $\begin{array}{l}F t(1,8,33,4)=7,8, p<0,001, \eta 2=0,29 \\
F t(2,0,38,2)=5,7, p<0,001, \eta 2=0,23\end{array}$ \\
\hline SDNN, ms & $\begin{array}{l}\text { T3 } \\
\text { T4 }\end{array}$ & $\begin{array}{l}33,06(14,80) \\
40,66(17,65)\end{array}$ & $\begin{array}{l}61,39(27,04) \\
59,23(23,19)\end{array}$ & $56,31(26,39)$ & $\begin{array}{l}60,04(29,51) \\
59,17(20,50)\end{array}$ & $\begin{array}{l}61,92(31,80) \\
60,66(20,69)\end{array}$ & $\begin{array}{l}57,01(29,96) \\
59,81(24,88)\end{array}$ & $\begin{array}{l}F t(2,3,44,3)=14,3, p<0,001, \eta 2=0,43 \\
F t(2,7,50,9)=11,1, p<0,001, \eta 2=0,37\end{array}$ \\
\hline$=\%$ & $\begin{array}{l}\text { T3 } \\
\text { T4 }\end{array}$ & $\begin{array}{l}63,38(19,11) \\
73,56(19,21)\end{array}$ & $\begin{array}{l}89,59(5,86) \\
86,73(9,64)\end{array}$ & $88,30(10,35)$ & $\begin{array}{l}89,99(5,36) \\
89,12(6,98)\end{array}$ & $\begin{array}{l}87,97(6,76) \\
90,52(6,25)\end{array}$ & $\begin{array}{l}85,41(9,81) \\
84,20(16,09)\end{array}$ & $\begin{array}{l}F t(2,1,39,7)=23,1, p<0,001, \eta 2=0,55 \\
F t(1,8,34,3)=8,1, p<0,001, \eta 2=0,30\end{array}$ \\
\hline
\end{tabular}

M, Mittelwert; SD, Standardabweichung; BL, Ruhemessung; BF-T, Biofeedback mit Taktgeber; BF-1, Biofeedback 1; BF-2, Biofeedback 2; BF-3, Biofeedback 3; SR, Selbstregulationsphase; T3, 3. Therapiesitzung; T4, 4. Therapiesitzung; SDNN, "standard deviation of the NN interval"; RMSSD, "root mean square of successive differences between NN interval"; LF, Low-frequency-Bereich.

Tabelle 2. Teststatistiken der Kontrastanalysen zur dritten und vierten HRV-BF-Sitzung $(n=20)$

\begin{tabular}{llll}
\hline & & Baseline vs. Biofeedback-Phasen & $\begin{array}{l}\text { Biofeedback-Phasen vs. } \\
\text { Selbstregulationsphase }\end{array}$ \\
\hline RMSSD, ms & T3 & $F t(1,19)=13,4, p<0,001, \eta 2=0,41$ & $F t(1,19)=0,09, p=0,77, \eta 2=0,00$ \\
& T4 & $F t(1,19)=26,9, p<0,001, \eta 2=0,59$ & $F t(1,19)=0,11, p=0,75, \eta 2=0,00$ \\
\hline SDNN, ms & T3 & $F t(1,19)=24,9, p<0,001, \eta 2=0,57$ & $F t(1,19)=0,57, p=0,45, \eta 2=0,03$ \\
& T4 & $F t(1,19)=27,2, p<0,001, \eta 2=0,59$ & $F t(1,19)=0,00, p=0,97, \eta 2=0,00$ \\
\hline LF, \% & T3 & $F t(1,19)=36,5, p<0,001, \eta 2=0,66$ & $F t(1,19)=4,38, p=0,05, \eta 2=0,19$ \\
& T4 & $F t(1,19)=14,49, p<0,001, \eta 2=0,43$ & $F t(1,19)=2,3, p=0,15, \eta 2=0,11$ \\
\hline
\end{tabular}

T3, 3. Therapiesitzung; T4, 4. Therapiesitzung; SDNN, "standard deviation of the NN interval"; RMSSD, "root mean square of successive differences between NN interval"; LF, Low-frequency-Bereich.

terschied von der Ruhemessung zu den Biofeedbackeinheiten (BF-T, BF-2 und BF-3) für die Parameter RMSSD, SDNN und LF ( $p$-Werte $<0,001)$ mit großen Effekten. Dies zeigt auch hier eine größere HRV während der Biofeedbackeinheiten im Vergleich zur Ruhemessung. Es ergab sich kein signifikanter Unterschied zwischen den Biofeedbackeinheiten (BF-T, BF-2 und BF-3) und der Selbstregulationsphase ( $p$-Werte $>0,05)$, während auch hier die Parameter eine größere HRV während der Selbstregulationsphase gegenüber der Ruhemessung abbilden. Abbildung 2a zeigt den Verlauf während der letzten Trainingssitzung für den Parameter RMSSD.

$H R V$-Analysen über die Sitzungen hinweg. Betrachtet man die Ruhemessungen zu den vier Trainingssitzungen und eine Woche nach dem Training im Verlauf, zeigte sich ein signifikanter großer Zeiteffekt für den HRV-Parameter SDNN $\left(F_{t}(4,80)=5,41, p<0,001, \eta^{2}\right.$ $=0,27)$ (Abb. 2b). Die Parameter RMSSD $\left(F_{t}(4,80)=\right.$ $\left.2,76, p=0,07, \eta^{2}=0,12\right)$ und $\operatorname{LF}\left(F_{t}(4,80)=1,78, p=\right.$ $\left.0,17, \eta^{2}=0,08\right)$ zeigten deskriptiv Anstiege im Mittel, jedoch keine signifikanten Zeiteffekte. Die Bonferroni korrigierten Post-hoc-Analysen ergaben eine signifi- kante Steigerung des SDNN von der Ruhemessung vor der ersten Sitzung (Mittelwert $=32,21, \mathrm{SD}=22,5$ ) zur Ruhemessung nach der vierten Sitzung (Mittelwert = $50,54, \mathrm{SD}=29,46)$ mit einem großen Effekt $(t(20)=$ $-3,63, p=0,002, d=-0,79$ ).

\section{Subjektive Anwendbarkeit - Akzeptanz}

Die Mehrheit der Teilnehmer*innen (90,9\%) gab an, dass sie zufrieden mit dem Biofeedbacktraining waren. Nur 13,6\% gaben milde Nebenwirkungen an, und eine Person berichtete von "ziemlichen" Nebenwirkungen. Betrachtet man die offenen Evaluationsangaben, wurde Schwindel während der Übung als Nebenwirkung von $n$ = 3 Personen angegeben. Andere Nebenwirkungen wurden nicht berichtet.

Abschließend gaben 59,1\% eine mindestens "ziemliche" Verbesserung des körperlichen Befindens und 63,6\% einen mindestens "ziemlich" verbesserten Umgang mit den körperlichen Beschwerden durch die BiofeedbackIntervention an (Abb. 3). Eine positive Auswirkung auf die Stimmung durch das Biofeedbacktraining berichteten $68,2 \%$ und eine positive Auswirkung auf das Stressemp- 
Abb. 2. Ergebnisse der HRV-Analysen zum Verlauf innerhalb einer Biofeedbacksitzung (a zeigt Mittelwerte und Standardabweichungen des RMSSD zur dritten Biofeedbacksitzung) und über die Intervention hinweg (b zeigt Mittelwerte und Standardabweichungen des SDNN der Baseline über die Sitzungen hinweg). BL, Baseline; BF-T, Biofeedback mit Taktgeber; BF-2, BF-3, Biofeedbackeinheiten 2 und 3 (mit Feedback); SR, Selbstregulationsphase.

Abb. 3. Subjektive Verbesserung im körperlichen Befinden und im Umgang mit den körperlichen Beschwerden durch HRV-BF nach Abschluss der Intervention (Angaben in relativen Häufigkeiten).
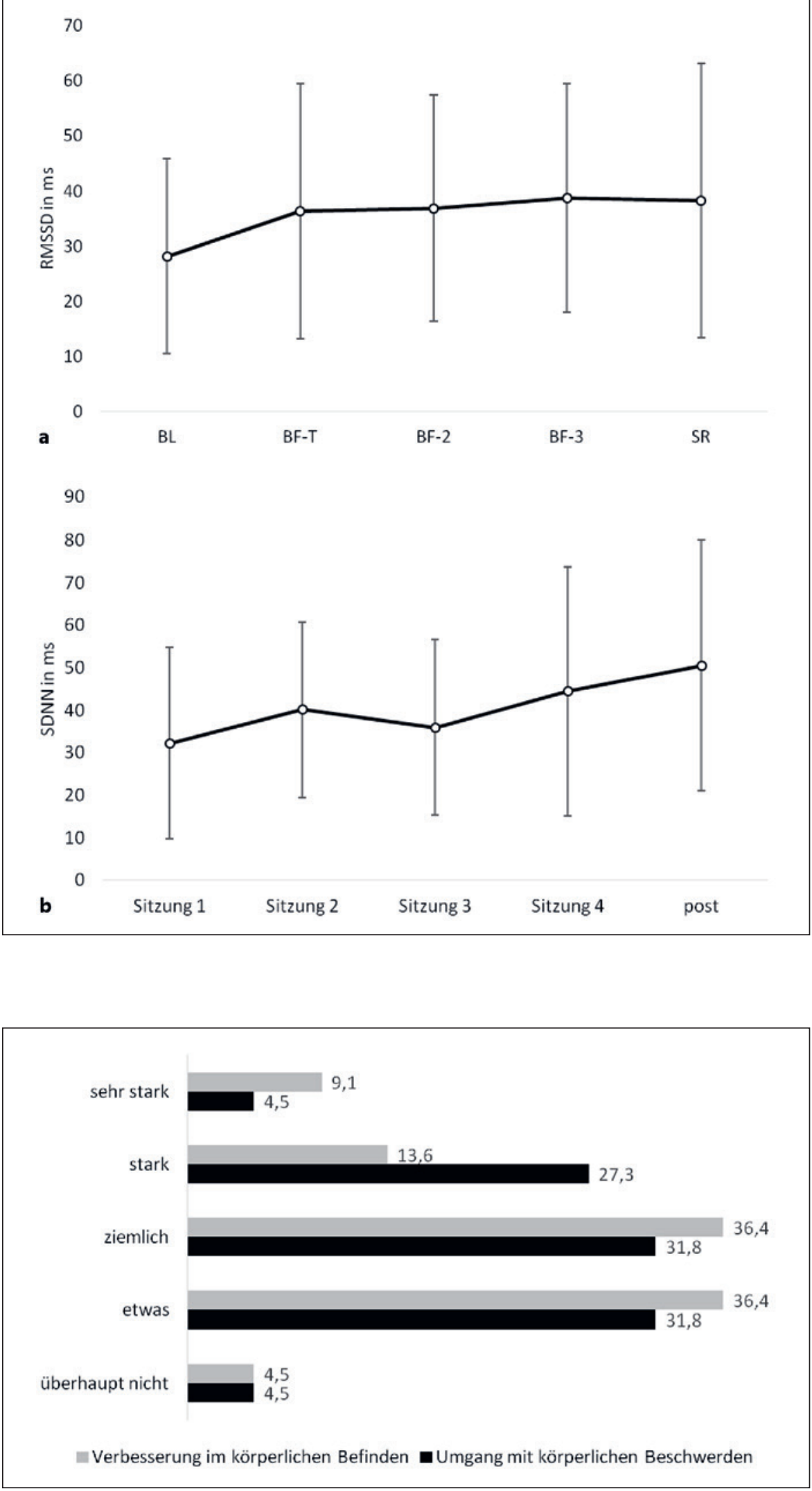
finden $72,7 \%$. Insgesamt 90,9\% der Teilnehmer*innen gaben an, dass sie das Training auch nach dem Studienende im Alltag fortführen wollen.

\section{Diskussion}

Die HRV als Maß der Funktionalität des autonomen Nervensystems scheint bei der SBS herabgesetzt zu sein [Cheng et al., 2020]. Das HRV-BF bietet hier als Intervention die Möglichkeit der direkten Stimulation des autonomen Nervensystems [Lehrer und Gevirtz, 2014]. Vor allem im Bereich der SBS erscheint die Weiterentwicklung psychologischer Interventionen aufgrund nur geringer Effektstärken notwendig. Ziel der Studie war es daher, die Anwendbarkeit einer neu entwickelten Kurzzeitintervention in Form eines HRV-BF zu überprüfen.

Die Ergebnisse zeigen erwartungsgemäß eine Veränderung der HRV-Parameter im Rahmen des Resonanzfrequenztrainings sowohl während als auch über die Sitzungen hinweg sowie eine hohe Zufriedenheit mit der vermittelten Technik. Die Personen waren innerhalb der Trainingssitzungen in der Lage, die Technik anzuwenden. Dies zeigte sich durch eine signifikante Steigerung der HRV-Parameter SDNN, RMSSD und LF von der Ruhemessung zu den Biofeedbackeinheiten mit großen Effektstärken. Der Anstieg des LF ist damit zu erklären, dass während einer sehr langsamen Atmung der Vagusnerv stimuliert wird [Kromenacker et al., 2018]. Außerdem ist eine Steigerung des LF mit einer Stimulierung des Baroreflexes assoziiert [Lehrer et al., 2003]. Wie von uns erwartet wurde, lernten die Personen, die Technik des RF-BF $\mathrm{zu}$ verinnerlichen und konnten diese in der Selbstregulationsphase aufrechterhalten. Dies zeigte sich durch nahezu unveränderte Werte in der HRV im Vergleich der Biofeedback-Phasen mit Rückmeldung und der Selbstregulationsphase, in der weder eine sichtbare Rückmeldung der HRV noch der Taktgeber als Hilfsmittel zur Atmungssteuerung gegeben waren. Die Daten weisen darauf hin, dass die Techniken der Selbstregulation bereits in der dritten Sitzung erlernt waren. Diese Ergebnisse stimmen mit den Ergebnissen von Lehrer et al. [2013] überein, dass die Selbstkontrolltechniken schnell erworben werden können. Bei den physiologischen Parametern zeigt sich über die Ruhemessungen hinweg noch kein einstimmiges Bild, da sich die Veränderung nur in einem Parameter (SDNN) statistisch signifikant zeigte. In dem weiteren Indikator (RMSSD) zeigte sich der Anstieg über die Sitzungen hinweg als Trend.

Hinsichtlich der subjektiven Rückmeldung der Personen ergab sich zusammenfassend eine hohe Zufriedenheit und Akzeptanz. Nach nur vier Sitzungen berichteten die Personen eine positive Auswirkung auf ihre Stimmung, das Stressempfinden sowie auf das körperliche
Empfinden, sowie dass sie einen besseren Umgang mit ihren körperlichen Symptomen erreicht hatten. Vor dem Hintergrund einer Stichprobe mit stark chronifizierten körperlichen Beschwerden ist dies beachtlich und deutet auf eine gute Wirksamkeit hin. Insgesamt ist Biofeedback eine Methode, die sehr gut von den Patient*innen akzeptiert wird [Rief und Birbaumer, 2011].

Die vorliegende Arbeit hat als erste eine Kurzintervention in Form eines HRV-BF bei Personen mit einer SBS als Teil einer randomisiert-kontrollierten Pilotstudie untersucht. Das Biofeedback kann schnell erlernt werden und zeigt zudem sowohl subjektive als auch physiologische Verbesserungen unmittelbar (eine Woche) nach Therapieende. Die hier präsentierten Ergebnisse zu den physiologischen Veränderungen zwischen einzelnen Behandlungssitzungen und -phasen basieren rein auf den Daten der Biofeedbackgruppe ohne Kontrollgruppe. Für die weiteren Ergebnisse, differenziert für verschiedene Bereiche der Symptomatik und der Ruhe-HRV von Therapiebeginn zu -ende im Vergleich zu einer alternativen aktiven Therapie, verweisen wir auf die Arbeit von Klewinghaus und Martin [eingereicht].

Durch das hier entwickelte Biofeedback-Manual kam es sowohl zu einer Symptomreduktion als auch Verbesserung der psychobehavioralen Komponenten (exzessive Gedanken, Gefühle, Verhalten) der SBS [Klewinghaus und Martin, 2021]. Die hier dargestellten Ergebnisse erlauben jedoch nicht zu beurteilen, ob die erzielten subjektiven oder physiologischen Effekte positiver ausfallen als bei der Anwendung einer anderen Intervention. Stärke der Studie ist die detaillierte Analyse der HRV in zeit- und frequenzbasierten Parametern mit sehr sorgfältiger Artefaktkontrolle. Zudem repräsentiert die Stichprobe das Krankheitsbild der SBS durch eine lange Beschwerdedauer sowie einer hohen Rate an Komorbiditäten gut. Neben den Stärken weist die Studie auch Limitationen auf. Auch wenn die Stichprobengröße im Vergleich zu vorherigen Studien groß ist, ist die statistische Power begrenzt. Somit konnte der Einfluss möglicher weiterer Variablen, die mit der HRV in Verbindung stehen, nicht kontrolliert werden. Demnach wären in Zukunft Studien mit größeren Stichproben wünschenswert. Zudem muss noch gezeigt werden, ob die Ergebnisse unmittelbar nach Therapieende auch nach längerer Anwendung in einer Nachbeobachtung stabil bleiben. Ermutigend ist, dass die Bereitschaft, das Training auch nach Studienende im Alltag fortführen zu wollen, mit 90,9\% Zustimmung sehr hoch liegt.

Gemäß den hier dargestellten Studienergebnissen, bietet das HRV-BF zum einen eine Möglichkeit des niederschwelligen Therapieangebots in der gestuften Versorgung und zum anderen eine Ergänzung zu einer Psychotherapie. Personen könnten orientiert an der S3Leitlinie für funktionelle Körperbeschwerden ein
Klewinghaus/Martin 
niederschwelliges Angebot in der erweiterten Versorgung erhalten, um eine weitere Chronifizierung zu minimieren und frühzeitig an den Beschwerden anzusetzen [AWMF, 2018]. Zudem bietet das Biofeedback in der multimodalen Behandlung eine Möglichkeit, Psychotherapie um die physiologischeEbenezu ergänzen. Vor allem Patient*innen, die ein sehr somatisch geprägtes Krankheitsverständnis haben, können so über das Biofeedback einen Zugang zur Psychotherapie erhalten [Nanke und Rief, 2004]. Caldwell und Steffen [2018] zeigten, dass die Hinzunahme von HRVBF zu einer Psychotherapie im Vergleich zu Psychotherapie alleine bei Personen mit einer "Major Depression" zu einer stärkeren Verbesserung in der Symptomatik und zu einem Anstieg in der HRV führte.

Ausgehend davon, dass die Personen eine positive Auswirkung auf ihre Stimmung und das Stressempfinden berichteten, lässt sich annehmen, dass das Biofeedbackprotokoll auch bei anderen Störungsbildern wie z.B. depressiven Störungen gut einsetzbar scheint. Dies zeigen auch bereits vorherige Studien, die eine gute Wirksamkeit auf die Depressivität durch HRV-BF nachweisen konnten [Karavidas et al., 2007; Pinter et al., 2019].

In Zukunft wird es interessant sein, den Mehrwert des Biofeedbacks bei der Ergänzung zur Psychotherapie zu untersuchen, so wie bei Caldwell und Steffen [2018] mit vielversprechenden Ergebnissen bei Personen mit "Major Depression".

\section{Hinweis}

Materialien zur Intervention (Informationsblätter und Protokollbogen) können bei den Autor*innen angefragt werden.

\section{Dank}

Wir danken MSc Louisa Langhoff, MSc Jana Doormann, MSc Elisabeth Kusber und BSc Ulrike Klau für ihre Hilfe bei der Rekrutierung und Durchführung der Studie.

\section{Ethische Aspekte}

Ein positives Ethikvotum der Ethikkommission der Bergischen Universität Wuppertal liegt vor (MS/BBL 190327). Die ethischen Standards der Deklaration von Helsinki in ihrer erweiterten Form wurden eingehalten. Die Patient*innen gaben ihre schriftliche Einwilligung zur Studienteilnahme und Publikation.

\section{Interessenskonflikt}

Die vorliegende Arbeit ist Teil der Dissertation von Laura Klewinghaus. Laura Klewinghaus erhielt Honorar für einen Workshop zum Biofeedback als Teil der postgradualen Weiterbildung in kognitiver Verhaltenstherapie. Alexandra Martin erhielt Honorare für Workshops zum Biofeedback und kognitiver Verhaltenstherapie bei somatoformen Störungen und als Editor der Zeitschrift Psychotherapeut.

\section{Finanzielle Unterstützung}

Die Forschung erhielt keine finanzielle Unterstützung.

\section{Autorenmitwirkung}

Die Konzeptualisierung der vorliegenden Studie erfolgte durch L.K. und A.M. L.K. analysierte und interpretierte die Ergebnisse. Alle Autorinnen diskutierten die Ergebnisse. L.K. erstellte das Manuskript mit Beiträgen und Überarbeitung von A.M.

\section{Datenverfügbarkeit}

Die Daten, auf die die Ergebnisse der Studie beruhen, können beim entsprechenden Autor mit Begründung angefragt werden.

\section{Literatur}

American Psychiatric Association (APA). Diagnostic and statistical manual of mental disorders. 5. Ausg. Washington: American Psychiatric Association; 2013.

Appelhans BM, Luecken LJ. Heart rate variability as an index of regulated emotional responding. Rev Gen Psychol. 2006 Aug;10(3):22940 .

Arbeitsgemeinschaft der Wissenschaftlichen Medizinischen Fachgesellschaften (AWMF). S3 Leitlinie "Funktionelle Körperbeschwerden”. Frankfurt: AWMF; 2018.

Beauchaine TP, Thayer JF. Heart rate variability as a transdiagnostic biomarker of psychopathology. Int J Psychophysiol. 2015 Aug;98: 338-50.
Caldwell YT, Steffen PR. Adding HRV biofeedback to psychotherapy increases heart rate variability and improves the treatment of major depressive disorder. Int J Psychophysiol. 2018;131:96-101.

Chalmers JA, Quintana DS, Abbott MJ, Kemp AH. Anxiety disorders are associated with reduced heart rate variability: a meta-analysis. Front Psychiatry. 2014 Jul;5:1-11.

Cheng YC, Huang YC, Huang WL. Heart rate variability in patients with somatic symptom disorders and functional somatic syndromes: a systematic review and meta-analysis. Neurosci Biobehav Rev. 2020 Feb;112:336-44.
Engel GL. The need for a new medical model: a challenge for biomedicine. Science. 1977;196: 129-36.

Hassett AL, Radvanski DC, Vaschillo EG, Vaschillo B, Sigal LH, Karavidas MK, et al. A pilot study of the efficacy of heart rate variability (HRV) biofeedback in patients with fibromyalgia. App Psychophysiol Biofeedback. 2007;32:1-10.

Herzog A, Shedden-Mora MC, Jordan P, Löwe B. Duration of untreated illness in patients with somatoform disorders. J Psychosom Res. 2018;107:1-6.

Holroyd KA, Penzien DB, Hursey DL, Tobin DL, Rogers L, Holm JE, et al. Change mechanisms in EMG biofeedback training: cognitive changes underlying improvements in tension headache. J Consult Clin Psychol. 1984;52:1039-53. 
Karavidas MK, Lehrer PM, Vaschillo E, Vaschillo $\mathrm{B}$, Marin $\mathrm{H}$, Buyske $\mathrm{S}$, et al. Preliminary results of an open label study of heart rate variability biofeedback for the treatment of major depression. Appl Psychophysiol Biofeedback. 2007 Mar;32:19-30.

Klewinghaus L, Martin A. Brief heart rate variability biofeedback treatment for somatic symptom disorder: results from a pilot randomized controlled trial. In: Löwe B, Martin A, Hrsg. Diagnosis and treatment options for somatic symptom disorders [symposium]. 8th Annual Conference of the European Association of Psychosomatic Medicine and CL Psychiatry, Virtual Conference Online. 2021.

Klewinghaus L, Martin A. Efficacy of heart rate variability biofeedback for somatic symptom disorder: a pilot randomized controlled trial. Eingereicht. 2021.

Koch C, Wilhelm M, Salzmann S, Rief W, Euteneuer F. A meta-analysis of heart rate variability in major depression. Psychol Med. 2019 May;49:1948-57.

Kromenacker BW, Sanova AA, Marcus FI, Allen JJB, Lane RD. Vagal mediation of low-frequency heart rate variability during slow yogic breathing. Psychosom Med. 2018 Aug;80: 581-7.

Lehrer PM. Heart rate variability biofeedback and other psychophysiological procedures as important elements in psychotherapy. Int J Psychophysiol. 2018 Sep;131:89-95.

Lehrer PM, Gevirtz R. Heart rate variability biofeedback: how and why does it work? Front Psychol. 2014 Jul;5:756-9.

Lehrer PM, Kaur K, Sharma A, Shah K, Huseby R, Bhavsar J, et al. Heart rate variability biofeedback improves emotional and physical health and performance: a systematic review and meta analysis. Appl Psychophysiol Biofeedback. 2020 May;45:109-29.

Lehrer PM, Vaschillo E, Vaschillo B. Resonant frequency biofeedback training to increase cardiac variability: rationale and manual for training. Appl Psychophysiol Biofeedback. 2000;25:177-91.

Lehrer PM, Vaschillo E, Vaschillo B, Lu SE, Eckberg DL, Edelberg R, et al. Heart rate variability biofeedback increases baroreflex gain and peak expiratory flow. Psychosom Med. 2003; 65:796-805.

Lehrer PM, Vaschillo B, Zucker T, Graves J, Katsamanis M, Aviles M, et al. Protocol for heart rate variability biofeedback training. Biofeedback. 2013;41:98-109.
Margraf J, Cwik JC. Mini-DIPS open access: Diagnostisches Kurzinterview bei psychischen Störungen. Bochum: Forschungs- und Behandlungszentrum für Psychische Gesundheit, Ruhr-Universität Bochum; 2017.

Martin A, Härter M, Henningsen P, Hiller W, Kröner-Herwig B, Rief W. Evidenzbasierte Leitlinie zur Psychotherapie Somatoformer Störungen und assoziierter Syndrome. Göttingen: Hogrefe; 2013.

Mather M, Thayer J. How heart rate variability affects emotion regulation brain networks. Curr Opin Behav Sci. 2018 Jan;19:98-104.

Nanke A, Rief W. Biofeedback in somatoform disorders and related syndromes. Curr Opin Psychiatry. 2004;17(2):133-8.

Pinter A, Szatmar S, Horvath T, Penzlin AI, Barlinn K, Siepmann M, et al. Cardiac dysautonomia in depression: heart rate variability biofeedback as a potential add-on therapy. Neuropsychiatr Dis Treat. 2019;15:1287-310.

Quintana DS, Guastella AJ, McGregor IS, Hickie IB, Kemp AH. Heart rate variability predicts alcohol craving in alcohol dependent outpatients: further evidence for HRV as a psychophysiological marker of self-regulation. Drug Alcohol Depend. 2013 Sep;132:395-8.

Rief W, Birbaumer N. Grundsätzliches zu Biofeedback. In: Rief W, Birbaumer N, Hrsg. Biofeedback : Grundlagen, Indikationen, Kommunikation, Vorgehen. Stuttgart: Schattauer; 2011. p. 1-7.

Rief W, Burton C, Frostholm L, Henningsen P, Kleinstäuber M, Kop WJ, et al. Core outcome domains for clinical trials on somatic symptom disorder, bodily distress disorder, and functional somatic syndromes: European network on somatic symptom disorders recommendations. Psychosom Med. 2017;79:100815.

Rief W, Hiller W. Das Screening für somatoforme Störungen (SOMS). 2., vollständig überarbeitete und neu normierte Auflage. Huber: Bern; 2008.

Ryan M, Gevirtz R. Biofeedback-based psychophysiological treatment in a primary care setting: an initial feasibility study. Appl Psychophysiol Biofeedback. 2004 Jun;29:79-93.

Sammito S, Thielmann B, Zimmermann P, Böckelmann I. Einfluss einer posttraumatischen Belastungsstörung auf die Herzfrequenzvariabilität als Marker des autonomen Nervensystems: eine systematische Literaturübersicht. Fortschr Neurol Psychiatr. 2015;83:307.
Schmidt JE, Joyner MJ, Tonyan HM, Reid KI, Hooten WM. Psychological and physiological correlates of a brief intervention to enhance self-regulation in patients with fibromyalgia. J Musculoskelet Pain. 2012;20:211-21.

Schmidt J, Martin A. Neurofeedback reduces overeating episodes in female restrained eaters: a randomized controlled pilot-study. Appl Psychophysiol Biofeedback. 2015;40: 283-95.

Schmidt J, Martin A. Herzratenvariabilitäts-Biofeedback in der klinischen Praxis: Grundlagen, Anwendung und Evidenz auf Basis eines systematischen Reviews. Psychotherapeut. 2017;62:498-506.

Schuman DL, Killian MO. Pilot study of a single session heart rate variability biofeedback intervention on veterans' posttraumatic stress symptoms. Appl Psychophysiol Biofeedback. 2019;44:9-20

Steffen PR, Austin T, Debarros A, Brown T. The impact of resonance frequency breathing on measures of heart rate variability, blood pressure, and mood. Front Public Health. 2017;5: $1-6$.

Tarvainen MP, Niskanen JP, Lipponen JA, RantaAho PO, Karjalainen PA. Kubios HRV: heart rate variability analysis software. Comput Methods Programs Biomed. 2014;113:21020.

Thayer JF, Åhs F, Fredrikson M, Sollers JJ 3rd, Wager TD. A meta-analysis of heart rate variability and neuroimaging studies: implications for heart rate variability as a marker of stress and health. Neurosci Biobehav Rev. 2012;36:747-56.

Toussaint A, Hüsing P, Kohlmann S, Löwe B. Detecting DSM-5 somatic symptom disorder: criterion validity of the patient health questionnaire-15 (PHQ-15) and the somatic symptom scale-8 (SSS-8) in combination with the somatic symptom disorder-B criteria scale (SSD-12). Psychol Med. 2019;50:324-33.

van Dessel N, den Boeft M, van der Wouden JC, Kleinstäuber $\mathrm{M}$, Leone SS, Terluin B, et al. Non-pharmacological interventions for somatoform disorders and medically unexplained physical symptoms (MUPS) in adults (review). Cochrane Database Syst Rev. 2014; 11:CD011142.

Windthorst P, Mazurak N, Kuske M, Hipp A, Giel $\mathrm{KE}$, Enck $\mathrm{P}$, et al. Heart rate variability biofeedback therapy and graded exercise training in management of chronic fatigue syndrome: an exploratory pilot study. J Psychosom Res. 2017;93:6-13. 